\title{
EXPONENTIAL DYNAMICAL LOCALIZATION FOR THE ALMOST MATHIEU OPERATOR
}

\author{
SVETLANA JITOMIRSKAYA AND HELGE KRÜGER
}

\begin{abstract}
We prove that the exponential moments of the position operator stay bounded for the supercritical almost Mathieu operator with Diophantine frequency.
\end{abstract}

\section{INTRODUCTION}

As pointed out in [10, the spectral property of Anderson localization of a selfadjoint operator $H$ does not let us conclude much about the behavior of the time evolution $\mathrm{e}^{-\mathrm{i} t H}$ 1], with dynamical localization-type conclusions requiring either additional or separate arguments. Various dynamical localization formulations have been suggested and proved, by different methods, for most popular models, particularly, for the Anderson model. Aside from uniform dynamical localization that happens rarely for ergodic families [10, 17, the strongest dynamical localization property is the exponential (in space) rate of decay of the expected overlap, that is

$$
\mathbb{E} \sup _{t \in \mathbb{R}}\left|\left\langle\delta_{k}, \mathrm{e}^{-\mathrm{i} t H} \delta_{\ell}\right\rangle\right| \leq C \mathrm{e}^{-\gamma|k-\ell|}
$$

A stronger, but equivalent in all known examples, formulation is (1.1) with $\mathrm{e}^{-\mathrm{i} t H}$ replaced with an arbitrary bounded function $f(H)$, or, equivalently, by the exponential decay of $\mathbb{E} \sum_{s}\left(\left|\varphi_{s}(0) \| \varphi_{s}(k)\right|\right)$, where $\left\{\varphi_{s}\right\}_{s}$ is a complete set of orthonormalized eigenfunctions (and the sum may be localized in energy, if needed). As pointed out in [3, this leads to various interesting physical conclusions, for example the exponential decay of the two-point function at the ground state and positive temperatures with correlation length staying uniformly bounded as temperature goes to zero. Moreover, it is this conclusion that is often implicitly assumed as manifesting localization, in physics literature. It is therefore desirable to establish it for physically relevant models.

It is natural in this context to define the exponential decay rate in expectation (obviously connected to the minimal inverse correlation length) as

$$
\gamma:=\liminf _{k \rightarrow \infty}\left(-\frac{\ln \mathbb{E}\left(\sum_{s}\left|\varphi_{s}(0)\right| \cdot\left|\varphi_{s}(k)\right|\right)}{|k|}\right)
$$

with a relevant question being whether or not it is positive. This definition can be localized to an energy range by summing over the eigenfunctions with energies

Date: August 14, 2012.

2000 Mathematics Subject Classification. Primary 81Q10; Secondary 37D25, 47B36.

Key words and phrases. Almost Mathieu operator, dynamical localization.

H. K. was supported by a fellowship of the Simons foundation.

${ }^{1}$ If $\psi(t)$ solves $\mathrm{i} \dot{\psi}=H \psi$, then $\psi(t)=\mathrm{e}^{-\mathrm{i} t H} \psi(0)$. 
falling in the range, in which case it is linked to the minimal inverse correlation length for Fermi energies falling in that range.

The corresponding question for the Anderson model, i.e. for the potential being independent identically distributed random variables, was solved in [12, 20, for the one-dimensional case and in 1, 4] for higher dimensions throughout the regimes where corresponding proofs of localization work, thus establishing positivity of $\gamma$.. The corresponding result for continuum operators was proven in [2].

In this paper we establish the first result of this type for a non-random ergodic operator 2 The most natural and popular operator that fits this description is the almost Mathieu operator given by

$$
\begin{aligned}
H_{\lambda, \alpha, \theta}: \ell^{2}(\mathbb{Z}) & \rightarrow \ell^{2}(\mathbb{Z}), \\
H_{\lambda, \alpha, \theta} u(n) & =u(n+1)+u(n-1)+2 \lambda \cos (2 \pi(n \alpha+\theta)) u(n),
\end{aligned}
$$

where $\lambda>0, \alpha$ is irrational, $\theta \in \mathbb{R}$, and $\ell^{2}(\mathbb{Z})$ denotes the space of square summable bi-infinite sequences. We will be interested in the supercritical regime that is $\lambda>1$, which is the regime of localization for almost every value of $(\alpha, \theta)$.

The frequency $\alpha$ is Diophantine if there exist $\kappa, \tau>0$ such that

$$
\|q \alpha\|=\operatorname{dist}(q \alpha, \mathbb{Z}) \geq \frac{\kappa}{q^{\tau}}
$$

for all integers $q \geq 1$. We will also use the notation $0 \leq \beta(\alpha):=\lim \sup -\frac{\ln \|q \alpha\|}{q}$, so $\beta(\alpha)=0$ for Diophantine $\alpha$. Frequencies $\alpha$ with small $\beta(\alpha)$ (with the quantifier depending on the context) are often called weakly Liouville. It was shown in [18 that for $\lambda>1$ and $\alpha$ Diophantine Anderson localization holds, that is for almost every $\theta \in \mathbb{R}$, the operator $H_{\lambda, \alpha, \theta}$ has pure point spectrum with exponentially decaying eigenfunctions. This has been extended to the weakly Liouville case in $[5]$.

As far as quantum dynamics goes, the following dynamical statements are known:

(i) Jitomirskaya and Last have shown in [19] that for $\lambda>\frac{15}{2}, \alpha$ Diophantine, and almost every $\theta$ we have

$$
\sup _{t \in \mathbb{R}}\left|\left\langle\delta_{k}, \mathrm{e}^{-\mathrm{i} t H_{\lambda, \alpha, \theta}} \delta_{\ell}\right\rangle\right| \leq C \mathrm{e}^{-\gamma|k-\ell|}
$$

where $\gamma>0$ is independent of $\theta, k$, and $\ell$, but $C>0$ is allowed to depend on $\theta$ and $\ell$, but is independent of $k$.

(ii) Germinet and Jitomirskaya have shown in [13] that for $\lambda>1$ and $\alpha$ Diophantine, we have that for every $q>1$

$$
\int_{0}^{1} \sup _{t} \sum_{n \in \mathbb{Z}}(1+|n|)^{q}\left|\left\langle\delta_{n}, \mathrm{e}^{-\mathrm{i} t H_{\lambda, \alpha, \theta}} \delta_{0}\right\rangle\right| d \theta<\infty .
$$

This property is called strong dynamical localization in [13] and a number of publications on the Anderson model and quasiperiodic operators. As the conclusion of Theorem 1.1 is stronger a better name might be polynomial dynamical localization in expectation.

(iii) It is known since [14, 17 that a Diophantine-type assumption is necessary even for spectral localization.

\footnotetext{
2 aside from families with uniform localization, where this conclusion holds trivially. It should be noted that the existing examples with uniform localization (e.g. 9]) are of a rather artificial nature and do not correspond to physical systems
} 
(iv) Bourgain and Jitomirskaya [8] have proven dynamical localization results of the type (ii) for more general quasi-periodic models.

In this note we prove the above described exponential rate of decay for supercritical almost Mathieu operator with Diophantine (and even weakly Liouville) frequencies:

Theorem 1.1. Let $\lambda>1$. Then there exists $\beta>0$ such that for $\alpha$ with $\beta(\alpha)<\beta$ we have $\gamma>0$ with $\gamma$ defined in (1.2).

This immediately implies

Corollary 1.2. Under the conditions of Theorem 1.1 there exists $\gamma>0$ and $C>0$ such that

$$
\int_{0}^{1} \sup _{t \in \mathbb{R}}\left|\left\langle\delta_{\ell}, \mathrm{e}^{-\mathrm{i} t H_{\lambda, \alpha, \theta}} \delta_{k}\right\rangle\right| d \theta \leq C \mathrm{e}^{-\gamma|k-\ell|}
$$

for all $k, \ell \in \mathbb{Z}$.

The conclusion of this corollary can be best called exponential dynamical localization in expectation. It is clear that this result implies both the results of 13 and 19. The proof of Theorem 1.1] is based on the results of [6]. As the bounds on eigenfunction decay obtained in [6] are nonquantitative also our bounds on $\gamma$ are.

It is an interesting and natural question if the limit in the right-hand side of (1.2) exists in general and if for one dimensional operators $\gamma$ can be equal to the minimal Lyapunov exponent as that is how the eigenfunctions often decay (for the Diophantine almost Mathieu case the almost Lyapunov decay of the eigenfunctions was shown in [18). We plan to investigate this question in a future work. It is also interesting to establish positive decay rate for other well-studied models with localization, for example, for other quasiperiodic operators in the regime of positive Lyapunov exponents.

The proof of Theorem 1.1 splits into two parts. In Section 2 we reduce the question to a question of decay estimates of eigenfunctions with a given localization center. As we hope that the results will be useful in other contexts, we formulate this section for general families of operators acting on $\ell^{2}\left(\mathbb{Z}^{d}\right)$. In Section 3 , we deduce the appropriate decay estimate from the results of [6].

\section{Reduction to a Question about eigenfunctions}

As the results in the following are valid for general families of operators, we state them in this generality. Let $\left\{H_{x}\right\}_{x \in X}$ be a family of self-adjoint operators on $\ell^{2}\left(\mathbb{Z}^{d}\right)$, and $(X, \mu)$ a probability space. Assume that for $\mu$ almost every $x \in X$ the spectrum of $H_{x}$ is pure point. Denote by $\varphi_{x ; s}$ an orthonormal basis of $\ell^{2}\left(\mathbb{Z}^{d}\right)$ consisting of eigenfunctions of $H_{x}$. For each $\varphi_{x ; s}$, we let $n_{x ; s} \in \mathbb{Z}^{d}$ be such that

$$
\left|\varphi_{x ; s}\left(n_{x ; s}\right)\right|=\left\|\varphi_{x ; s}\right\|_{\ell \infty}\left(\mathbb{Z}^{d}\right) \text {. }
$$

It is shown for $d=1$ in Section 2 of [15]. that $\varphi_{x ; s}$ and $n_{x ; s}$ can be chosen to be measurable functions of $x$. The general case can be found in [16].

Fixing $\lambda>1$ and $\alpha$ with $\beta(\alpha)$ sufficiently small, the results of $[18$ imply that the family of operators $\left\{H_{\lambda, \alpha, \theta}\right\}_{\theta \in[0,1]}$ satisfy the above properties when $[0,1]$ is equipped with the normalized Lebesgue measure. 
We state the next theorem in a form more general than is necessary for application to the almost Mathieu operator. It is our hope that it will be useful in other contexts. The main difference to a more naive estimate is that one can exploit the orthogonality of the $\varphi_{x ; s}$ when estimating $\sum_{n_{x ; s}=n}\left|\varphi_{x ; s}(\ell)\right|^{2}$.

Theorem 2.1. We have for almost every $x$ that

$$
\begin{aligned}
\left|\left\langle\delta_{k}, \mathrm{e}^{-\mathrm{i} t H_{x}} \delta_{\ell}\right\rangle\right| & \leq \sum_{s}\left|\overline{\varphi_{x ; s}(k)} \varphi_{x ; s}(\ell)\right| \\
& \leq \sum_{n}\left(\sum_{n_{x ; s}=n}\left|\overline{\varphi_{x ; s}(k)}\right|^{2} \sum_{n_{x ; s}=n}\left|\varphi_{x ; s}(\ell)\right|^{2}\right)^{\frac{1}{2}}
\end{aligned}
$$

and

$$
\begin{aligned}
\int\left|\left\langle\delta_{k}, \mathrm{e}^{-\mathrm{i} t H_{x}} \delta_{\ell}\right\rangle\right| d \mu(x) & \leq \int \sum_{s}\left|\overline{\varphi_{x ; s}(k)} \varphi_{x ; s}(\ell)\right| d \mu(x) \\
& \leq \sum_{n}\left(\int \sum_{n_{x ; s}=n}\left|\varphi_{x ; s}(k)\right|^{2} d \mu(x) \int \sum_{n_{x ; s}=n}\left|\varphi_{x ; s}(\ell)\right|^{2} d \mu(x)\right)^{\frac{1}{2}} .
\end{aligned}
$$

Proof. Denote by $E_{x ; s}$ the eigenvalues of $H_{x}$ such that $H_{x} \varphi_{x ; s}=E_{x ; s} \varphi_{x ; s}$. We have that

$$
\left\langle\delta_{k}, \mathrm{e}^{-\mathrm{i} t H_{x}} \delta_{\ell}\right\rangle=\sum_{s} \overline{\varphi_{x ; s}(k)} \varphi_{x ; s}(\ell) \mathrm{e}^{-i t E_{x ; s}} .
$$

By the triangle inequality and reordering the sum, we obtain

$$
\left|\left\langle\delta_{k}, \mathrm{e}^{-\mathrm{i} t H_{x}} \delta_{\ell}\right\rangle\right| \leq \sum_{n \in \mathbb{Z}} \sum_{n_{x ; s}=n}\left|\overline{\varphi_{x ; s}(k)} \varphi_{x ; s}(\ell)\right| .
$$

The first equation follows by applying the Cauchy-Schwarz inequality to the inner sum. The second equation follows from the first by integrating and then applying the Cauchy-Schwarz to the terms of the form

$$
\int\left(\sum_{n_{x ; s}=n}\left|\overline{\varphi_{x ; s}(k)}\right|^{2}\right)^{\frac{1}{2}}\left(\sum_{n_{x ; s}=n}\left|\varphi_{x ; s}(\ell)\right|^{2}\right)^{\frac{1}{2}} d \mu(x) .
$$

Corollary 2.2. For $C, \gamma>0$ assume for $n, \ell \in \mathbb{Z}^{d}$, that

$$
\int \sum_{n_{x ; s}=n}\left|\varphi_{x ; s}(\ell)\right|^{2} d \mu(x) \leq C \mathrm{e}^{-2 \gamma|n-\ell|}
$$

Then

$$
\int\left|\left\langle\delta_{k}, \mathrm{e}^{-\mathrm{i} t H_{x}} \delta_{\ell}\right\rangle\right| d \mu(x) \leq \begin{cases}C_{1}(\gamma, d, C)\left(1+|k-\ell|^{d-1}\right) \mathrm{e}^{-\gamma|k-\ell|}, & d>1 \\ C\left(\frac{1+\gamma}{\gamma}+|k-\ell|\right) \mathrm{e}^{-\gamma|k-\ell|}, & d=1\end{cases}
$$




\section{Almost localization}

The goal of this section is to discuss consequences of the work [6] and to give the proof of Theorem 1.1 .

Definition 3.1. Let $\theta \in \mathbb{R}, k \in \mathbb{Z}$, and $\eta>0$. Then $k$ is called $\eta$-resonant for $\theta$ if

$$
\|2 \theta-k \alpha\| \leq \mathrm{e}^{-\eta|k|} .
$$

Given $\theta$ and $\eta$, we denote by $k_{j}$ the set of $\eta$-resonances for $\theta$.

Recall furthermore, that the Almost-Mathieu equation is given by

$$
h_{\lambda, \alpha, \theta} u(n)=u(n+1)+u(n-1)+2 \lambda \cos (2 \pi(n \alpha+\theta)) u(n) .
$$

We use the notation $h_{\lambda, \alpha, \theta}$ in contrast with that of (1.3) to emphasize that this is meant as a difference equation. Theorem 5.1. in [6] states that

Theorem 3.2. For $\lambda, C_{0}>1$ there exists $\eta(\lambda)>0$ and $\beta\left(\lambda, C_{0}\right)>0$ so that for $\alpha$ with $\beta(\alpha)<\beta$ there exist $C_{1}, \gamma>0$, such that for $\theta \in \mathbb{R}$ and any solution $u$ of $h_{\lambda, \alpha, \theta} u=E u$ with $u(0)=1$ and $|u(n)| \leq 1+|n|$, we have

$$
|u(n)| \leq C_{1} \mathrm{e}^{-\gamma|n|}
$$

for $C_{0}\left(1+\left|k_{j}\right|\right) \leq|n| \leq \frac{1}{C_{0}}\left|k_{j+1}\right|$ where $k_{j}$ denote the $\eta$-resonances of $\theta$.

This theorem implies

Theorem 3.3. We have that

$$
\int_{0}^{1}\left(\sum_{n=n_{\theta ; s}}\left|\varphi_{\theta ; s}(\ell)\right|^{2}\right) d \theta \leq\left(C_{1}\right)^{2} \mathrm{e}^{-2 \gamma|n-\ell|}+\mathrm{e}^{-\frac{\eta}{C_{0}}|n-\ell|} .
$$

Proof. By replacing $\theta$ by $\theta-n \alpha$ and $\ell$ by $\ell-n$, we can assume that $n=0$. Then for $n_{\theta ; s}=0$ the functions

$$
u_{s}=\frac{1}{\left|\varphi_{\theta ; s}(0)\right|} \varphi_{\theta ; s}
$$

satisfy the assumptions of the previous theorem. So we obtain that if $\ell$ is not $\eta$-resonant for $\theta$ that

$$
\left|\varphi_{\theta ; s}(\ell)\right| \leq C_{1}\left|\varphi_{\theta ; s}(0)\right| \mathrm{e}^{-\gamma|\ell|} .
$$

By orthogonality $\sum_{s}\left|\varphi_{\theta ; s}(0)\right|^{2}=1$, thus we obtain for these $\theta$ that

$$
\sum_{n_{\theta ; s}=0}\left|\varphi_{\theta ; s}(\ell)\right|^{2} \leq\left(C_{1}\right)^{2} \mathrm{e}^{-2 \gamma|\ell|}
$$

Hence, we obtain that

$$
\begin{aligned}
\int_{0}^{1} & \left(\sum_{n_{\theta ; s}=0}\left|\varphi_{\theta ; s}(\ell)\right|^{2}\right) d \theta \leq\left(C_{1}\right)^{2} \mathrm{e}^{-2 \gamma|\ell|} \\
& +\mid\left\{\theta: \quad \exists k \eta \text {-resonances of } \theta \text { with } \frac{1}{C_{0}}|k| \leq|\ell| \leq C_{0}(1+|k|)\right\} \mid .
\end{aligned}
$$

For fixed $k$ the set of $\theta$ such that $k$ is $\eta$-resonant for $\theta$ has measure $\mathrm{e}^{-\eta|k|}$. As the smallest possible choice for $k$ in the previous equation is $\frac{1}{C_{0}}|\ell|$ the claim follows.

Proof of Theorem 1.1 and Corollary 1.2. By the previous theorem, the assumptions of Corollary 2.2 hold. The claim follows. 


\section{REFERENCES}

[1] M. Aizenman, Localization at weak disorder: Some elementary bounds. Rev. Math. Phys. 6, 1163 (1994).

[2] M. Aizenman, A. Elgart, S. Naboko, F. Schenker, G. Stolz, Moment analysis for localization in random Schrdinger operators. Invent. Math. 163:2 (2006), 343-413.

[3] M. Aizenman, G.-M. Graf, Localization bounds for an electron gas. 1998 J. Phys. A: Math. Gen.31 (1998) 6783-6806.

[4] M. Aizenman, J. Schenker, R. Friedrich, D. Hundertmark, Finite-volume fractional-moment criteria for Anderson localization. Comm. Math. Phys. 224:1 (2001), 219-253.

[5] A. Avila, S. Jitomirskaya, The Ten Martini problem. Ann. of Math. 170, 303-342 (2009).

[6] A. Avila, S. Jitomirskaya, Almost localization and almost reducibility. JEMS 12 (2010), 93-131.

[7] Avron, J., Simon, B.: Singular continuous spectrum for a class of almost periodic Jacobi matrices. Bull. AMS 6, 81-85 (1982).

[8] J. Bourgain, S. Jitomirskaya, Anderson localization for the band model. GAFA, 6779, Lecture Notes in Math., 1745, Springer, Berlin, 2000.

[9] D. Damanik, Z. Gan, Limit-periodic Schrdinger operators with uniformly localized eigenfunctions. J. d'Analyse Math. 115 (2011), 33-49.

[10] R. Del Rio, S. Jitomirskaya, Y. Last, B. Simon, What is localization?. Phys. Rev. Lett. 75 (1995) 117-119.

[11] R. Del Rio, S. Jitomirskaya, Y. Last, B. Simon, Operators with singular continuous spectrum IV: Hausdorff dimensions, rank one pertubations and localization. J. d'Analyse Math. 69 (1996) 153-200.

[12] F. Delyon, H. Kunz, B. Souillard, One-dimensional wave equations in disordered media. J. Phys. A 16:1 (1983), 25-42.

[13] F. Germinet, S. Jitomirskaya, Strong dynamical localization for the almost Mathieu model. Rev. Math. Phys. 13:6 (2001), 755-765.

[14] Gordon, A.: On the point spectrum of the one-dimensional Schrödinger operator. Usp. Math. Nauk. 31, 257 (1976).

[15] A. Gordon, S. Jitomirskaya, Y. Last, B. Simon, Duality and singular continuous spectrum in the almost Mathieu equation. Acta Math. 178 (1997), 169-183.

[16] A. Gordon, A. Kechris, Measurable enumeration of eigenelements. Appl. Anal. 71:1-4 (1999), 41-61.

[17] S. Jitomirskaya, Continuous Spectrum and Uniform Localization for Ergodic Schrodinger Operators. J. Funct. Anal. 145, 312-322 (1997).

[18] S. Jitomirskaya, Metal-insulator transition for the almost Mathieu operator. Ann. of Math. (2) 150:3 (1999), 1159-1175.

[19] S. Jitomirskaya, Y. Last, Anderson localization for the almost Mathieu equation. III. Semiuniform localization, continuity of gaps, and measure of the spectrum. Comm. Math. Phys. 195:1 (1998), 1-14.

[20] H. Kunz, B. Souillard, Sur le spectre des oprateurs aux différences finies aléatoires. (French) [On the spectra of random finite difference operators]. Comm. Math. Phys. 78:2 (1980/81), 201-246,

University of California, Irvine, CA 92717

E-mail address: szhitomi@math.uci.edu

Mathematics 253-37, Caltech, Pasadena, CA 91125

E-mail address: helge@caltech.edu

$U R L:$ http://www.its.caltech.edu/ ${ }^{\sim}$ helge/ 